\title{
Illumination Source Identification Using a CMOS Optical Microsystem
}

\author{
Ger de Graaf and Reinoud F. Wolffenbuttel
}

\begin{abstract}
An integrated silicon color sensor system has been designed and fabricated in CMOS without extra masks for color filtering. The application is in low-cost measurement of ambient light conditions, e.g., for display control in portable instruments, or for testing the intensity and spectral distribution of light sources. Signals proportional to the intensity and the spectral distribution of the incident light are available at the output in the form of two bit-streams. It is demonstrated that daylight can be distinguished from other light sources, such as incandescent lamps and fluorescent lamps.
\end{abstract}

Index Terms-CMOS optical sensor, color measurement, sigmadelta converter.

\section{INTRODUCTION}

C OLOR sensing is usually associated with imaging sensors composed of large arrays of photodetectors covered with a pattern of differently dyed color filters. In standard IC technology, readout circuits are also integrated. Many practical applications do merely require only a single-point color measurement. Although the image device itself is low-cost, the image information is highly superfluous in many single-point color measurement applications, and extracting the color information from the video signals requires advanced and complex data processing. The measurement of the spectral distribution of an illuminating light source is important, as changes can significantly affect or distort the human perception. A notorious example is the effect of an artificial light source in the perceived perception of the color of food on display. Another application is in the ability to distinguish between illumination by an artificial light source or by daylight, thus enabling the detection whether the system, in which the sensor is integrated, is being used indoors or outdoors. Implementation of a single-point color sensor enables the optimum readout of a display in a scientific instrument, but can also be of interest in a consumer product such as a portable phone.

The economic viability of a single-point color measurement system, considering the cost and availability of color imaging sensors in high volumes, critically depends on the possibility of realizing the system in a standard microelectronic process (preferably CMOS). Also adding the system to other functions on a single chip means that the system would have to fit in an unaltered CMOS process, using the layers available only and without additional compatible process steps. Fortunately, silicon IC processes do offer opportunities because a) the absorption

Manuscript received June 15, 2002; revised October 15, 2003.

The authors are with the Department for Microelectronics, Delft University

of Technology, Delft, The Netherlands (e-mail: G.deGraaf@its.tudelft.nl).

Digital Object Identifier 10.1109/TIM.2003.822476
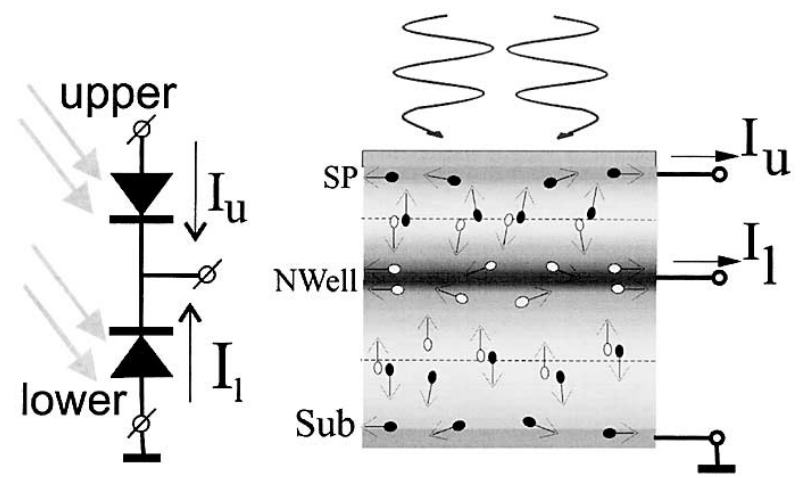

Fig. 1. Stacked photodetectors in silicon.

of light in silicon in the visible part of the spectrum is wavelength dependent [1], and b) the surface thin film layers can be used to form an interference filter with a peak transmission at a wavelength set by design and determined by layer thickness and material properties. Exploitation of these effects has been investigated by authors for many years [2], [3] and has actually also been proposed for the realization of a color imaging sensor [4], and it has recently also been applied in digital cameras [5]. In this paper, we concentrate on the design, fabrication, and performance measurement of CMOS integrated color measuring systems for the identification of a set of artificial light sources and daylight.

\section{SENSOR OPERATION}

There are several approaches for changing the spectral response of photodetectors fabricated in a CMOS process without the use of extra masks or processing steps. If PN junctions at different depths in the silicon can be fabricated, as shown in Fig. 1, these devices can have a significantly different spectral responsivity. This effect is due to the strong wavelength dependence of the absorption coefficient shown in Fig. 2 [1], which causes the penetration depth of light in silicon $d(\lambda)=1 / \alpha(\lambda)$ to be wavelength dependent. Incident photons with a wavelength of about $400 \mathrm{~nm}$ (blue) are absorbed within a layer up to $0.1 \mu \mathrm{m}$ below the silicon surface, whereas red light at $600 \mathrm{~nm}$ penetrates about $10 \mu \mathrm{m}$ into the silicon, as described in [3]. As electron-hole pairs are generated upon absorption, the vertical distribution of these generated charge carriers is penetration depth dependent. The vertically stacked junctions are used for depth-dependent collection of this photocharge and thus yield photocurrents $\left(\mathrm{I}_{\mathrm{u}}, \mathrm{I}_{\mathrm{l}}\right)$ depending on junction depth. An extension of this method is indicated in Fig. 3 [3] and basically includes a technique for more clearly and more flexibly assigning an absorption volume 


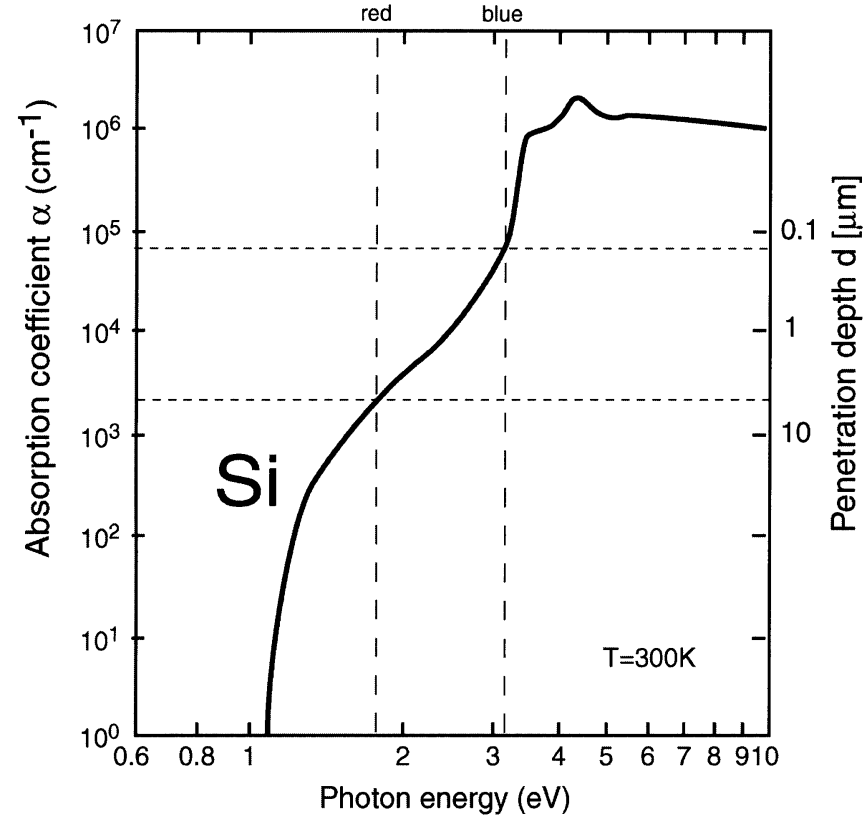

Fig. 2. Absorption coefficient and penetration depth of light in silicon.

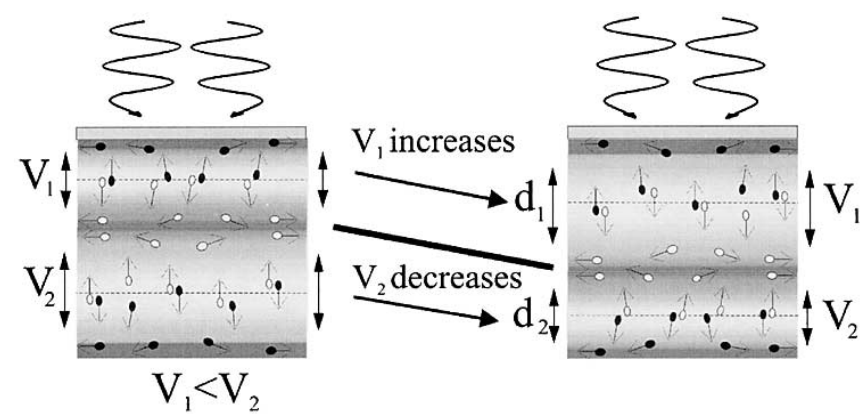

Fig. 3. Modulation of the depletion width $\left(\mathrm{d}_{1}, \mathrm{~d}_{2}\right)$ of stacked PN junctions.

to a collecting junction by avoidance of a neutral layer in between. The width of the depletion layers $\left(\mathrm{d}_{1}, \mathrm{~d}_{2}\right)$ increases with reverse voltages $\left(\mathrm{V}_{1}, \mathrm{~V}_{2}\right)$ applied across the stacked junctions and, therefore, the collection volumes of both PN junctions can be tuned by their reverse voltages. As can be seen from the absorption coefficient curve of silicon (Fig. 2), significant changes in responses can be obtained if the depletion layers can be varied in the order of magnitude of $1 \mu \mathrm{m}$ or more in the region between 360 and $500 \mathrm{~nm}$. This can be achieved in layers with relatively low carrier concentration $\left(<10^{15} / \mathrm{cm}^{-3}\right)$. In most CMOS processes, these layers are not available, therefore this voltage control effect is rather small, but it can be used for fine tuning of the spectral response.

The alternative property of a CMOS process, that can be used by designers to achieve a large differences in spectral response of integrated photodiodes, is the fabrication of several diodes of equal area, each covered with different dielectric of (semi-)conducting layers. The difference in optical path for impinging light results in interference peaking at a different wavelength. The thickness and the optical properties of the layers that are used have to be stable and well known for all batches of the standard process [6]. Since technology rules also have to be met, the

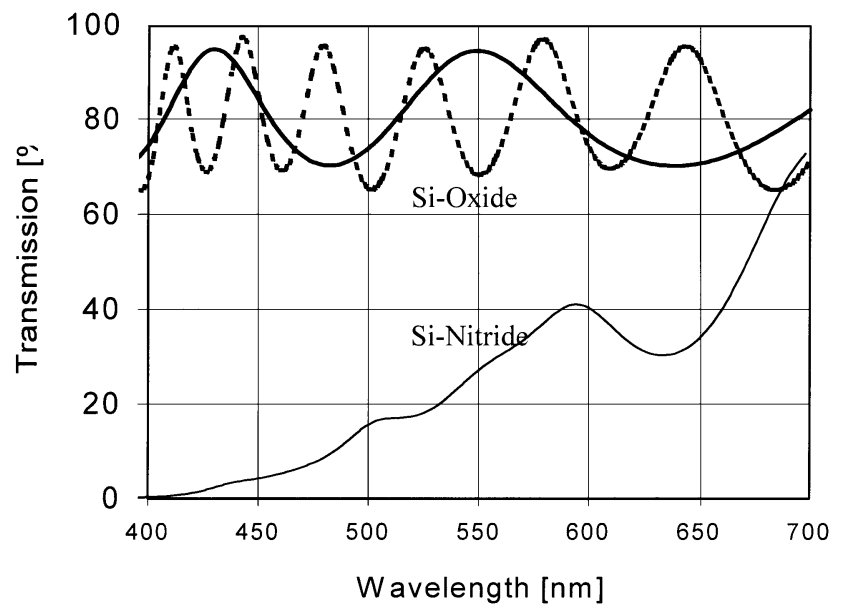

Fig. 4. Optical transmission of layers above the silicon.

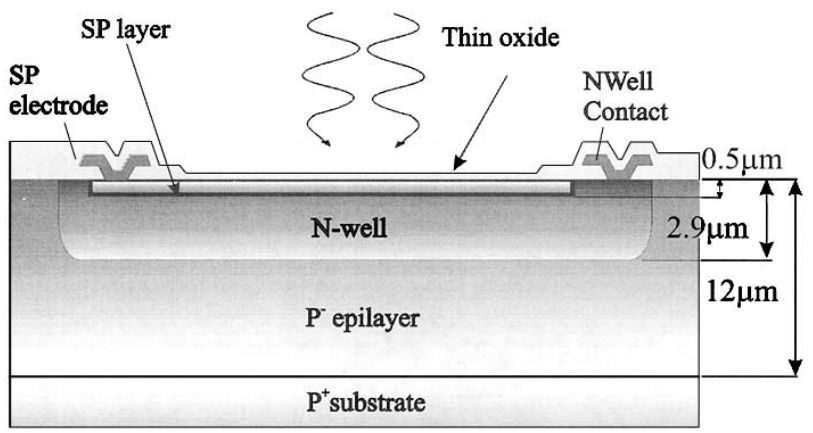

Fig. 5. Cross section of the fabricated CMOS color sensor.

design of the optical path is restricted to combinations of a limited amount of suitable layers above the PN junctions. The maximum obtainable spectral selectivity, therefore, depends highly on the process used. In Fig. 4 simulations using a thin-film calculation program [7] of the optical transmission of some typical combinations of layers are shown. The two top curves indicate that combinations of dielectric layers, like BPSG and oxy-nitride, can reduce the surface reflectance from $70 \%$, in the case of a direct air-silicon optical interface, to about $20 \%$, but also introduces a wavelength dependence. The lower curve shows the effect of a thin poly-silicon layer on top of the surface, absorbing almost all short-wavelength light.

\section{SENSOR FABRICATION}

The area of the photodiode is $0.48 \mathrm{~mm}^{2}$. The basic structure of the CMOS compatible sensor is shown in Fig. 5. Basically, two vertically stacked PN junctions, available in a standard CMOS process, are used. The shallow junction is between the $\mathrm{P}^{+}$-implanted layer, which is used for the drain and source layer, and the $\mathrm{N}$-well. The deeper junction is between the $\mathrm{N}$-well and the P-epilayer. The upper absorption volume $\left(\mathrm{P}^{+}-\mathrm{N}\right.$-well junction) contributes to the shallow junction photocurrent and the lower absorption volume ( $\mathrm{N}$-well-P-epilayer junction) to that of the lower diode. As impinging blue/UV light is already fully absorbed within a depth less than $0.5 \mu \mathrm{m}$, such short wavelength light contributes to the photocurrent of the upper junction only. Similarly, longer wavelength light 


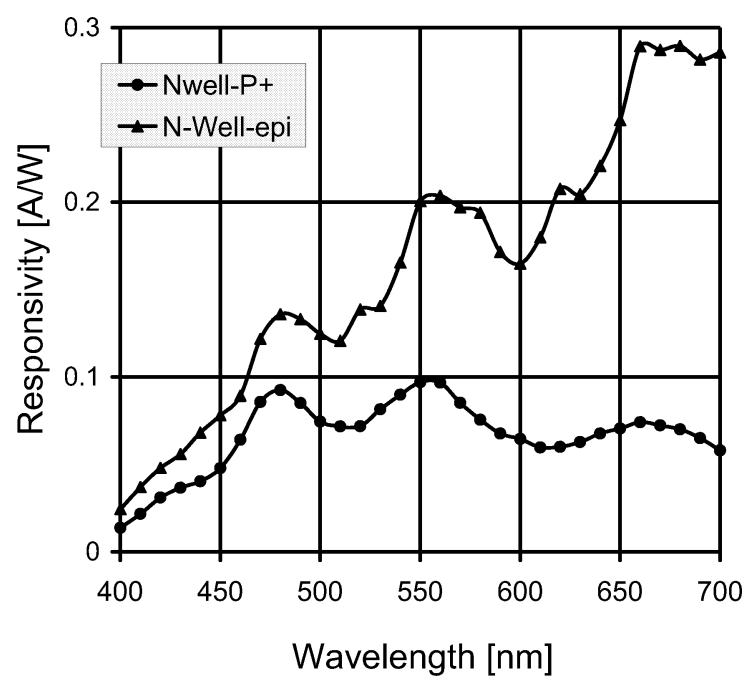

Fig. 6. Spectral responses of the fabricated device.

generates predominantly photocharge at larger depths and, thus, contributes mainly to the lower-junction photocurrent. The BPSG oxide thickness above the diodes is measured as $650 \mathrm{~nm}$, the thickness of the SP layer is $0.5 \mu \mathrm{m}$, and the depth of the N-Well is $2.8 \mu \mathrm{m}$. Fig. 6 shows the spectral response curves of the fabricated photodiodes, measured using a SPEX /Jobin-Yvon Triax-180 monochromator, a Xenon lamp light source, and a calibrated Hamamatsu S1200 photodiode as a reference. Clearly, the deep junction exhibits a better long-wavelength response as compared to the shallow junction.

\section{SENSOR REAdOUT}

The $\mathrm{N}$-well contact supplies both the $\mathrm{P}^{+}$-layer-N-well photocurrent $I_{u}$ plus the N-well-P-epilayer photocurrent $I_{l}$, and, thus, is a good measure for the total incident photon flux. The photocurrent measured at this terminal is, therefore, the intensity signal. The ratio of the photocurrent supplied at the drain/source contact divided by the $\mathrm{N}$-well current $\eta=\left(\mathrm{I}_{\mathrm{u}} / \mathrm{I}_{\mathrm{u}}+\mathrm{I}_{\mathrm{l}}\right)$ is in first order approximation independent of the light intensity due to the high linearity of both the photodetectors and the readout methods presented here. Therefore, this ratio is a good measure for the spectral information and is referred to as the color signal. The measured color signal of the sensor $\eta_{\mathrm{i}}(\lambda)=\mathrm{I}_{\mathrm{u}} / \mathrm{I}_{\mathrm{u}}+\mathrm{I}_{\mathrm{l}}$ is shown in Fig. 7. Several circuits are suitable for readout of $I_{u} / I_{u}+I_{1}$. A very simple realization is based on a first-order oscillator A-to-D conversion, as shown in Fig. 8. This circuit has been described in [8]. The dominant time constant of this circuit is determined by the photodetector itself. The circuit provides a digital output signal with a frequency proportional to the light intensity and a duty-cycle proportional to the color signal. The method described here is applying the Sigma-Delta $(\Sigma-\Delta) \mathrm{AD}$ conversion principle shown in Fig. 9 for the readout. The oversampling principle allows a flexible tradeoff between bandwidth and accuracy (noise). The reverse biased junction capacitance of the photodiodes and capacitor $\mathrm{C}_{\mathrm{fb}}$ are used as a storage element, integrating the photocurrent. The photocurrent discharges the initial charge across the photodetector and lowers the voltage at the input of the comparator. If this voltage level drops below

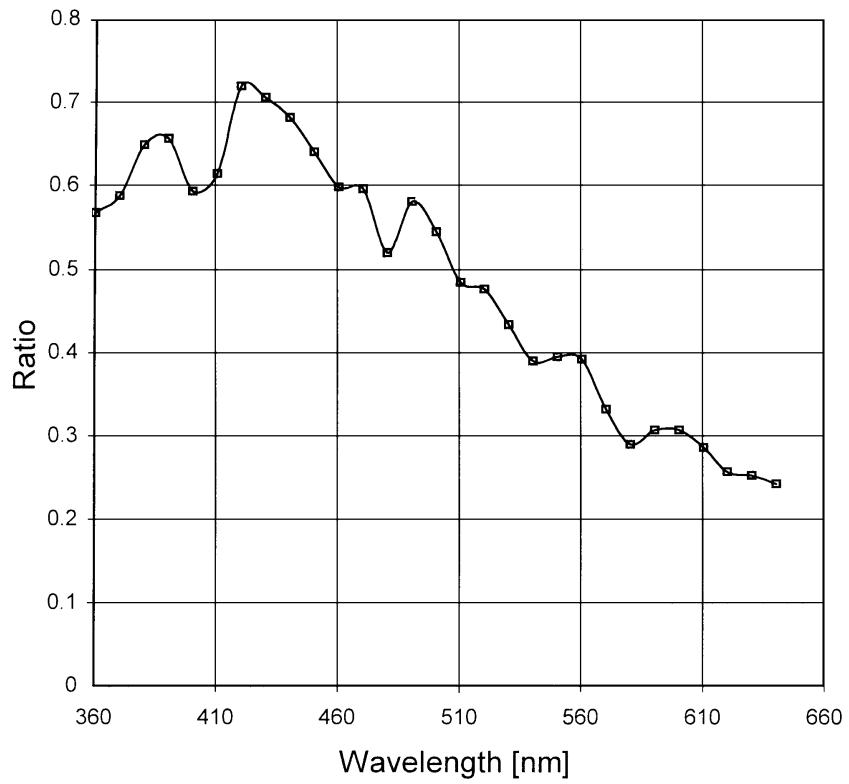

Fig. 7. Ratio of the photocurrents as a function of the wavelength.

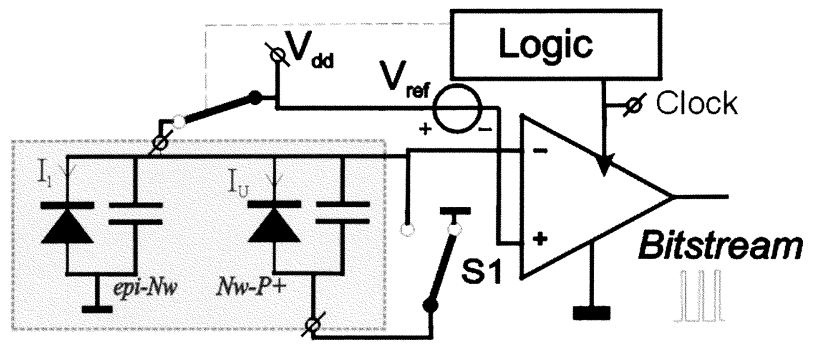

Fig. 8. Color sensor in a first order oscillator circuit.

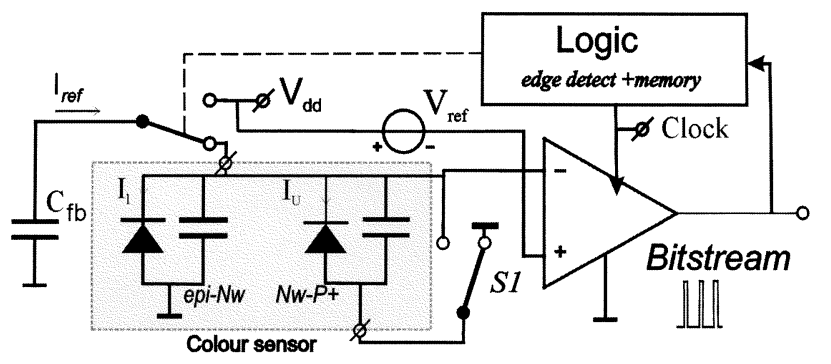

Fig. 9. Readout of the color sensor using a charge mode $\Sigma-\Delta$ AD converter.

the reference level $V_{r}$, capacitor $C_{f b}$ will be switched to $V_{d}$ during one clock cycle and during the subsequent clock cycles a charge $\mathrm{Q}_{\mathrm{fb}}=\left(\mathrm{V}_{\mathrm{dd}}\left(\mathrm{V}_{\mathrm{dd}}-\mathrm{V}_{\mathrm{ref}}\right)\right) \mathrm{C}_{\mathrm{fb}}=\mathrm{V}_{\text {ref }} \mathrm{C}_{\mathrm{fb}}$ is added to the sensor. The voltage across the sensor will therefore increase again and the comparator input voltage will oscillate around $\mathrm{V}_{\mathrm{r}}$. The charge-loss $\mathrm{Q}_{\mathrm{ph}}$ due to the photocurrent $\mathrm{I}_{\mathrm{photo}}$ between two output pulses equals $Q_{p h}=I_{\text {photo }} T_{\text {bitstream }}$. The average bitstream output frequency therefore simply equals $\mathrm{f}_{\text {bitstream }}=\mathrm{I}_{\text {photo }} / \mathrm{V}_{\mathrm{ref}} \mathrm{C}_{\mathrm{fb}}$ and can be scaled with the value of the charge injection capacitor $\mathrm{C}_{\mathrm{fb}}$. Two conversion cycles are needed to measure both signals. In the first phase, $I_{u}$ plus $I_{1}$ are measured (both junctions parallel) and during the second phase only the current provided by the lower junction $\mathrm{I}_{1}$ is monitored (upper junction shorted). Two 16-bit up/down counters are used to integrate the bit-streams at the output of the comparator and 


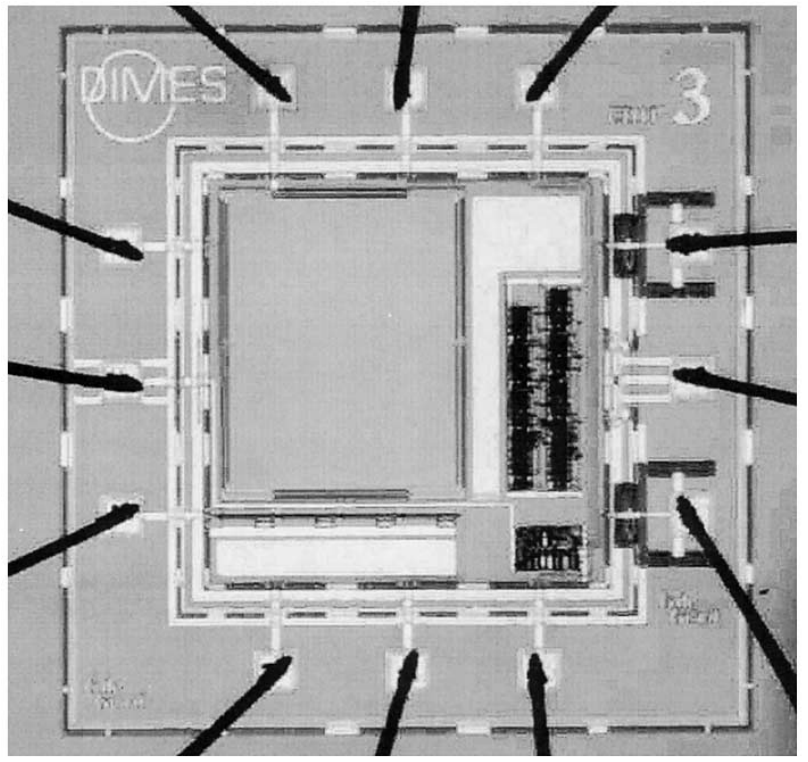

Fig. 10. Microphotograph op the optical microsystem.

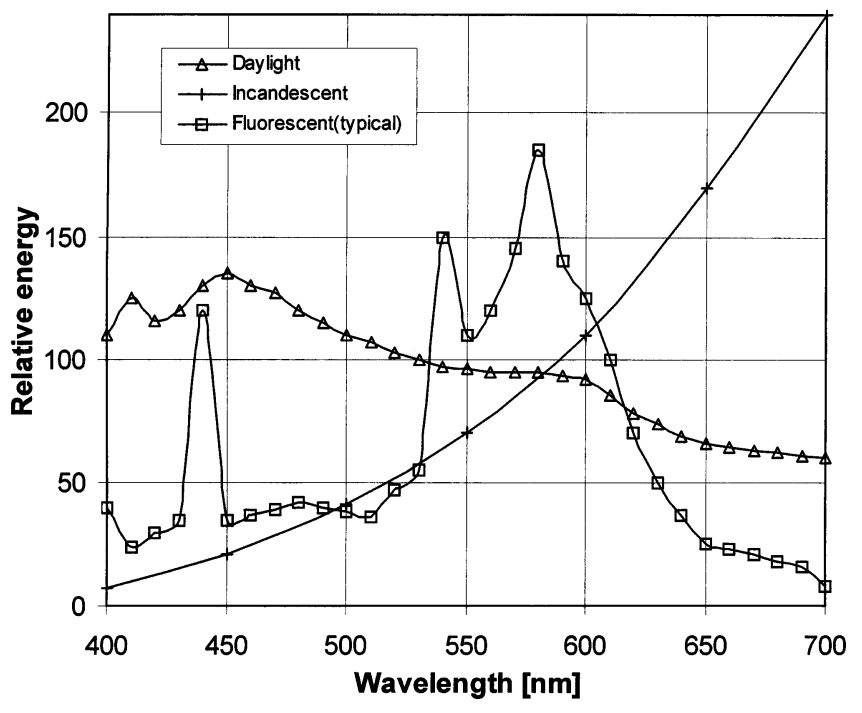

Fig. 11. Typical spectra of three different illumination sources.

store the values of the photocurrent from the lower and upper junction. From these values, the intensity and color signal can easily be calculated. A microphotograph of the realized system in CMOS is shown in Fig. 10.

\section{LIGHT SOURCE IDENTIFICATION}

Low-cost identification of environmental light conditions is needed in many portable consumer products in order to distinguish between indoor and outdoor mode of operation. This color sensor is very suitable in such applications. Fig. 11 shows the typical spectral distributions $\mathrm{P}(\lambda)$ of incandescent-, fluorescent- and daylight. Multiplication of these spectra with the spectral sensitivity of the sensor from Fig. 7 yields the spectral response of the ratio $\eta_{\text {calc }}=\mathrm{I}_{\mathrm{u}} / \mathrm{I}_{\mathrm{u}}+\mathrm{I}_{\mathrm{l}}$. Averaging these values over the visible spectrum, and introducing a scale factor $c_{r}$ for the readout circuit, the ratio $\mathrm{N}_{\text {freq }}$ of the bitstream frequencies of the lower and upper junction can be calculated:
TABLE I

Calculated and Measured OutPut Frequency Ratios

\begin{tabular}{c|c|c}
\hline & Calculated & Measured \\
Light Source & $\mathrm{N}_{\text {freq }}$ & $\mathrm{N}_{\mathrm{bs}}$ \\
\hline Incandescent & 0.76 & 0.735 \\
\hline Fluorescent & 0.72 & -- \\
Fluorescent (cold) & 0.71 & 0.73 \\
\hline Daylight & & \\
(clouded sky) & 0.685 & 0.70 \\
\hline
\end{tabular}

$N_{\text {freq }}=c_{\mathrm{r}} \int_{\lambda=\lambda_{\min }}^{\lambda=\lambda_{\max }} \eta_{\mathrm{i}}(\lambda) P(\lambda) d \lambda$. Table I compares the calculated values of $\mathrm{N}_{\text {freq }}$ with the measured ratio $\mathrm{N}_{\mathrm{bs}}=\mathrm{f}_{\mathrm{u}} / \mathrm{f}_{\mathrm{l}}+\mathrm{f}_{\mathrm{u}}$ of the bitstream frequencies for the three illumination conditions.

\section{CONCLUSION}

The CMOS integrated optical microsystem presented here enables the measurement of intensity and the average spectral content of impinging light without the need for extra masks for color filtering. This is an important advantage over other color measurement systems. The relatively simple readout circuits and the compliance with a standard CMOS process allow addition of this optical microsystem to an existing CMOS design, lowering the cost of the total system. The cost comparison is with the CCD camera. It should be noted that the present die is relatively large, and cost is mainly determined by the optical package. The photodiode area can be reduced, and modern CMOS processes allow a further reduction of the size of the circuits, making a more economically viable die. Long integration times allow an effective suppression of noise, and, therefore, in many applications, the photodiodes can be smaller as the ones presented here. In the case of a stand-alone system, the bit-stream outputs and on-chip counters facilitate direct interfacing. The sensors have to be optimized for a large difference in spectral response in a given CMOS process. This can be achieved by selecting junctions with a large difference in depth in the silicon, but also by using the optical properties of the various dielectric layers above the diodes and the package. Especially in the blue/UV range, the optical path requires special attention and can increase or decrease the spectral sensitivity. Light intensity is typically measured with a four-decade dynamic range. The output frequencies of the bit-streams are proportional to the optical intensity and range between $0.3 \mathrm{~Hz}$ and $100 \mathrm{kHz}$. The frequency ratio of both bit-streams is due to the spectral content and varies with a maximum of about $9 \%$ from incandescent light to direct daylight. This frequency ratio is in first-order approximation independent of the light intensity. Unlike conventional color measurement systems, in which three different spectral responses are measured, the operating mechanism for measuring spectral content in this sensor is based on 
bi-chromatic sensing. As a result, the spectral selectivity is inherently limited. Nevertheless, the system can distinguish between incandescent light and daylight. Unambiguous identification of fluorescent light from daylight conditions can be realized if the ac component in the optical signal is also detected.

\section{ACKNOWLEDGMENT}

The authors would like to thank the IC processing section of the Delft Institute for Microelectronics and Submicrontechnology (DIMES), Delft, The Netherlands, for the fabrication of the photodiodes and circuits.

\section{REFERENCES}

[1] H. R. Philip and E. A. Taft, "Optical constants of silicon in the region 1 to $10 \mathrm{eV}$," Phys. Rev., vol. 120, pp. 37-38, 1960.

[2] R. F. Wolffenbuttel, "Method and Circuit for Determining the Wavelength of Light," U.S.Patent 4749 851, Aug. 29, 1986.

[3] —_ "Silicon photodetectors with a selective spectral response," in Sensors Update, H. Baltes, J. Hesse, and J. Korvink, Eds. New York: Wiley, 2001, vol. 9, pp. 69-101.

[4] J. S. Hayward, B. B. Snavely, and P. S. Chan, "Color Image Sensing," European Patent 0015711 , Febr. 27, 1980.

[5] Foveon X3 Image Sensors. Foveon Inc, Santa Clara, CA. [Online]. Available: www.foveon.com

[6] D. P. Poenar and R. F. Wolffenbuttel, "Optical properties of thin-film silicon-compatible materials," Appl. Opt., vol. 36, no. 21, pp. 5122-5128, July 1997.
[7] “TFCALC 3.31," Software Spectra Inc., Portland, OR.

[8] G. de Graaf, F. Riedijk, and R. F. Wolffenbuttel, "Color sensor system with a frequency output and an ISS or $\mathrm{IC}^{2}$ bus interface," Sens. Actuators A, vol. 61, pp. 441-445, 1997.

[9] G. de Graaf and R. F. Wolffenbuttel, "Smart optical sensor system in CMOS for measuring light intensity and color," Sens. Actuators A, vol. 67, pp. 115-119, 1998.

Ger de Graaf received the B.S.E.E. degree in electrical and control engineering from the Technische Hogeschool, Rotterdam, The Netherlands, in 1983.

Since 1992, he has had a consultancy company specializing in electronic design and computer-controlled measurement systems. He is currently working on electronic circuits for silicon sensors.

Reinoud F. Wolffenbuttel received the M.Sc. and Ph.D. degrees from the Delft University of Technology (DUT), Delft, The Netherlands, in 1984 and 1988, respectively.

Between 1986 and 1993, he was an Assistant Professor and since 1993, he has been an Associate Professor with the Department of Microelectronics, Faculty of Information Technology and Systems, DUT, and is involved in instrumentation and measurement in general and on-chip functional integration of microelectronic circuits and silicon sensors, IC process compatible MEMS fabrication, and silicon microsystems, in particular. He was a Visitor at the University of Michigan, Ann Arbor, in 1992, 1999, and 2001, Tohoku University, Sendai, Japan, in 1995, and EPFL, Lausanne, Switzerland, in 1997.

Dr. Wolffenbuttel is the recipient of a 1997 NWO pioneer award. He served as the General Chairman of the Dutch National Sensor Conference in 1996, Eurosensors in 1999, and the MicroMechanics Europe Workshop in 2003. 